\title{
Kernos
}

Revue internationale et pluridisciplinaire de religion grecque antique

18 | 2005

Varia

\section{Antje KOLDE, Politique et religion chez Isyllos d'Épidaure}

Pierre Sineux

(2) OpenEdition

Journals

Édition électronique

URL : http://journals.openedition.org/kernos/1546

DOI : 10.4000/kernos. 1546

ISSN : 2034-7871

\section{Éditeur}

Centre international d'étude de la religion grecque antique

Édition imprimée

Date de publication : 1 janvier 2005

Pagination : 538-542

ISSN : 0776-3824

Référence électronique

Pierre Sineux, «Antje KoLdE, Politique et religion chez Isyllos d'Épidaure », Kernos [En ligne], 18 | 2005, mis en ligne le 24 mai 2011, consulté le 21 septembre 2020. URL : http://journals.openedition.org/ kernos/1546; DOI : https://doi.org/10.4000/kernos.1546 
Antje Kolde, Politique et religion chez Isyllos d'Épidaure, Basel, Schwabe \& CO AG Verlag, 2003. 1 vol. $16 \times 23$ cm, XIV + 443 p. (Schweizerische Beiträge zur Altertumswissenschaft, 28). ISBN : 3-7965-2000-6.

Isyllos d'Épidaure n'est connu que par une inscription de 79 lignes, datant du début du III $^{e}$ siècle av. J.-C. et découverte dans le sanctuaire d'Asklépios d'Épidaure en 1885 par P. Kavvadias. Ce citoyen d'Épidaure avait fait graver une stèle, placée bien en vue, qui se présentait comme une dédicace à Apollon Maléatas et à Asklépios et par laquelle il faisait connaitre successivement une nouvelle loi cultuelle qu'il avait fait établir en l'honneur des divinités, un péan, et le récit d'un «miracle » : Isyllos, alors qu'il était enfant et malade, avait vu le dieu Asklépios lui annoncer son intervention à Sparte pour lutter contre Philippe; et il s'était alors fait le messager de la nouvelle auprès des Lacédémoniens. L'editio princeps de ce texte composite fut établie l'année même de sa découverte et fut suivie d'une série d'éditions (seize au total) qui s'est achevée en 1929 avec celle de F. Hiller von Gaertringen (IG IV, 12, 128). La seule monographie existante sur Isyllos était celle de U. von Wilamowitz parue en 1886 (Isyllos von Epidauros, Berlin). Autant dire que l'ouvrage d'A. Kolde, issue d'une thèse de doctorat légèrement modifiée soutenue à l'Université de Genève en juillet 2001, veut combler une lacune et montrer que ce texte, tout en étant porteur d'une tradition littéraire, est ancré dans un contexte politique et religieux précis.

Les deux premiers chapitres, qui occupent plus de 220 pages (1. L'inscription d'Isyllos; 2. Commentaire), constituent en quelque sorte le socle de l'ouvrage. Après des rappels sur la découverte et les éditions antérieures, une description précise de la pierre, l'A. établit une nouvelle édition accompagnée d'une traduction soignée, dont les partis pris sont justifiés dans le commentaire qui suit. La structure générale du texte est ensuite dégagée et l'A. montre comment les sept «segments» de l'inscription, rendus visibles par le lapicide qui avait gravé des paragraphoi grammai dans la marge gauche, peuvent être reliés les uns aux autres, de sorte qu'apparaît une construction en trois parties : la première partie, composée des deux premiers segments, fournit des éléments sur le contexte de l'offrande faite à Apollon Maléatas et à Asklépios; vient ensuite le segment occupé par la nouvelle loi cultuelle instaurée par Isyllos; la dernière partie forme un triptyque ordonné autour du péan en l'honneur d'Apollon et d'Asklépios : ce péan est précédé de quelques lignes en prose relatives à la consultation de la Pythie et d'un petit poème sur l'origine du culte d'Apollon Maléatas puis il est suivi du récit du "miracle». Après l'analyse métrique des parties versifiées (les segments 3, 4 et 7 sont dans un mètre dactylique, le segment 2 en mètre trochaïque et le segment 6 , correspondant au péan, en mètre ionique), l'A. organise un commentaire découpé selon les sept segments de l'inscription et dans lequel on trouve pour chacun d'entre eux une analyse de la prosodie, une étude de la structure et des éléments dialectaux, ainsi qu'une succession de mises au point et de discussions sur les termes et les expressions utilisées. L'ensemble, étayé par une bibliographie solide, est impossible à résumer, mais nous pouvons retenir des développements très utiles, comme par exemple ceux qui concernent Isyllos et son identité de citoyen épidaurien (p. 43-48), Maléatas (p. 49-52 et 117-118), les notions telles que andragathia (p. 57-60) - même si la traduction par «vertu virile » n'est pas forcément la plus adéquate pour exprimer la qualité de l'anèr agathos (p. 59) -, aidôs (p. 76-79), terme difficile à traduire comme l'A. le montre bien (le mot «respect» retenu en définitive affaiblit quelque peu le sens), kalokagathia (p. 89-95), eunomia (p. 97-102). Pour l'étude de ces termes, grand profit aurait pu être tiré de l'ouvrage d'A. Fouchard, Aristocratie et démocratie. Idéologies et sociétés en Grèce ancienne (Besançon, 1997). On trouve 
également dans ce chapitre une bonne définition du "péan » (p. 135-140), suivie d'une bonne analyse de la structure et d'une étude judicieuse de la répartition des noms propres. Au sujet du troisième segment se référant à la loi cultuelle proposée par Isyllos (avec institution d'une procession réservée à ceux qui auront été proclamés pour leur arétè et leur aidôs, et d'une prière), le détour par treize autres lois cultuelles (relatives à une fondation cultuelle et comportant le règlement de cette fondation) n'était sans doute pas nécessaire pour aboutir à la conclusion que le texte d'Isyllos contient les mêmes points que ceux que l'on rencontre habituellement dans les lois sacrées, à quelques différences près : ces éléments sont «dilués » dans tout le texte (c'est-à-dire qu'ils ne se limitent pas au seul segment se rapportant à la loi cultuelle) tandis que la «mise en forme littéraire» du texte fait que le vocabulaire et les expressions utilisés ne sont pas conformes à l'usage (p. 112-113). On trouve également un long développement à propos du vers 30 où l'on peut lire, à propos du sanctuaire

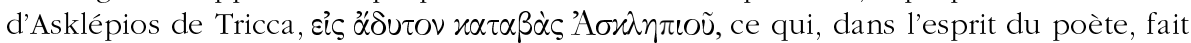
référence à un édifice analogue à un édifice épidaurien comme le oủó́ du début du vers 29 l'indique sans équivoque; l'A. développe l'idée selon laquelle adyton ne saurait être synonyme d'abaton mais doit correspondre, à Épidaure, à la partie souterraine de la tholos qui, selon l'hypothèse développée par R. Martin et H. Metzger (La religion grecque, Paris, 1976, p. 103-107), était notamment destinée à rappeler les pratiques oraculaires primitives par l'eau et le lien unissant Asklépios à Apollon. A ce sujet, disons que la discussion ne saurait être close. Il n'est pas si aisé de rejeter l'idée selon laquelle adyton pourrait être équivalent à abaton, comme de nombreux auteurs l'ont écrit (voir E.J. et L. Edelstein, Asclepius. A collection and interpretation of the testimonies, Baltimore, 1945, II, p. 191) et comme le laisse penser, au demeurant, la définition d'adyton que l'A. propose : «endroit clos, dans lequel seules certaines personnes ont le droit de pénétrer, à savoir des prêtres ou des gens qui s'y sont longuement préparés» (p. 120); l'abaton peut se définir de la même manière. En outre, le terme est bien attesté à Lébéna dans le même sens qu'abaton (comme il est rappelé p. 121) et si, à Épidaure, sur la stèle B, au début de la 1. 113, on ne lit en effet que les lettres SYTOr là où l'on attend le génitif d'abaton, on ne voit pas comment on pourrait

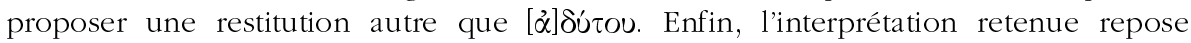
essentiellement sur l'idée générale que la descente vers un bassin ou une source dissimulée devait être indispensable aux rites asklépieiens (p. 125, n. 604); cette idée se fonde, en partie au moins, sur les conclusions de R. Ginouvès qui aurait dû être cité ici (Balaneutikè. Recherches sur le bain dans l'Antiquité grecque, 1962 [BEFAR, 200] et "L'eau dans les sanctuaires médicaux », in L'eau, la santé et la maladie dans le monde grec, 1994 [BCH, Suppl. 28], p. 237-246), mais il convient de rappeler aussi que cette lecture procède d'une interprétation des données archéologiques et qu'aucun texte ne vient formellement rendre compte directement de cette pratique. Ces remarques n'enlèvent rien à la qualité générale du commentaire détaillé.

La deuxième partie de l'ouvrage s'ouvre sur un chapitre (chapitre 3) intitulé «Isyllos, le poète, la poétique et le genre littéraire du texte » (p. 223-256). L'A. tente de montrer qu'Isyllos devait appartenir à une famille «noble» d'Épidaure et qu'il était bien lui-même le poète, auteur du péan intégré à l'inscription, cherchant à se poser en digne successeur de Lycurgue, tant du point de vue «technique» (il promulgue une loi) que "politique» (ses convictions et l'esprit de la loi sont aristocratiques et se rattachent à Sparte). L'étude de la poétique et du genre littéraire montre bien comment Isyllos inscrit son texte dans un ensemble de traditions : les cinq segments versifiés appartiennent au genre de l'élégie pour le deuxième et le troisième, des épigrammes pour le quatrième, de la lyrique chorale pour le sixième, du récit épique pour le 
septième; le dialecte est dorien mais on trouve une proportion plus ou moins grande de dorismes selon les passages et les genres rencontrés; le niveau de langue est également soigneusement adapté à la nature des segments, émaillés de citations qui posent la question, abordée avec prudence, de l'intertextualité. Cette imprégnation d'une tradition culturelle n'empêche pas une certaine liberté formelle de s'exercer comme on peut l'observer, par exemple, dans l'utilisation de termes rares. En définitive, Isyllos, sans appartenir pour autant au courant des poetae docti de l'époque hellénistique, fait figure de poète érudit mais, en dépit de ses prétentions littéraires, le contenu du texte apparaît typique de l'historiographie locale, à visée essentiellement politique.

L'A. revient ensuite (chapitre 4) sur la question de la datation. Le point de départ en est le vers 58-59 où Isyllos mentionne, au début du récit final de l'inscription, l'intervention armée de Philippe contre Sparte, au devant de laquelle Asklépios serait allé avant de revenir à Épidaure pour guérir l'enfant Isyllos. La plupart des commentateurs avaient considéré qu'il s'agissait de l'invasion de la Laconie par Philippe II de Macédoine à la fin de l'automne 338, ce qui conduisait à dater l'inscription du début du III $^{e}$ siècle. Sans contester ce point de vue, l'A. entreprend de défendre la validité d'une hypothèse, qui avait déjà reçu quelque faveur, selon laquelle le Philippe mentionné serait Philippe Arrhidée, le demi-frère d'Alexandre, devenu roi à la mort de ce dernier sous le nom de Philippe III; l'épisode de l'intervention contre Sparte se placerait après la mort d'Antipatros en 319, quand Polyperchon cherchait l'appui des Grecs du continent pour faire face à la coalition formée par Cassandre, Antigone, Ptolémée et Lysimaque. Dans ce contexte, il aurait poussé Philippe à restaurer la ligue de Corinthe, ce qui aurait conduit Sparte à réactiver sa politique anti-macédonienne, au moment où les Arcadiens et surtout les Messéniens s'alliaient avec la Macédoine, comme le montre le traité conclu en 317 (connu par une stèle découverte en 1991) à propos de l'installation d'une garnison macédonienne sur le Mont Ithôme. L'événement auquel Isyllos fait allusion daterait alors de 317-316. L'A. ne tranche pas mais tente ensuite de montrer que l'inscription doit, en définitive, dater de 280, ce qui conviendrait mieux à l'hypothèse en faveur de Philippe III (Isyllos serait, dans ce cas de figure, à un âge «plus normal » [p. 264] au moment où il fait graver l'inscription). Pour étayer cette idée, l'A. établit une relation entre l'inscription d'Isyllos et l'offensive (connue par Justin) menée par le roi Areus pendant l'été 280 en Phocide contre les Étoliens, alliés d'Antigone Gonatas, et au cours de laquelle "presque toutes les villes de la Grèce » auraient participé à la guerre. Selon elle, en effet, Isyllos appellerait à la lutte contre les « Macédoniens », c'est-à-dire, en fait, contre tous ceux qui tentent d'asseoir leur pouvoir sur l'héritage d'Alexandre.

Pour convaincre, l'A. passe par une série de développements connexes à son sujet. Elle reprend la question du "mirage spartiate » afin de montrer qu'Areus avait fondé son expédition sur une certaine image de Sparte dont Isyllos se ferait le relais comme l'indiqueraient les mentions de la kalokagathia, de l'eunomia, les allusions à l'eirènè ou encore l'utilisation du terme ploutos, jugé récurrent dans les réflexions relatives à sa constitution. Ensuite, elle voit dans le récit du «miracle»plusieurs références aux guerres médiques (parallèles lexicaux, parallèles de situation : le dieu annonce son aide militaire, un message est confié à une personne à laquelle le dieu apparaît) dont on connaît la prégnance dans bon nombre de textes et de discours de l'époque classique, en particulier athéniens (au premier rang desquels le genre de l'epitaphios logos); elle établit alors une série de parallèles (Isyllos et Philippidès, Asklépios et Pan, la victoire des Spartiates sur Philippe II ou III et Marathon...) destinés à rendre le texte « lisible» par un public athénien autant que spartiate. L’inscription est alors comprise 
comme un appel à soutenir le projet d'Areus, adressé aux Spartiates comme aux Athéniens, en rappelant leur combat contre un ennemi commun et l'aide divine dont ils ont bénéficié dans le passé. C'est cette aide qu'il est prêt à leur garantir en raison du secours d'Asklépios aux Spartiates dont il fut le témoin dans son enfance. L'A. souligne alors la portée politique de l'inscription dont le contenu semblait à première vue essentiellement religieux (p. 290-291). Enfin, l'A. propose une réflexion basée sur l'hypothèse, non démontrée, qu'il existerait un "lien» entre l'épithète Sôter par laquelle Asklépios est honoré à Sparte et les Soteria de Delphes commémorant la victoire remportée sur les Galates en 279 par des Phocidiens et des Étoliens assistés d'une coalition de Grecs, victoire à laquelle a contribué Apollon, secondé par d'autres divinités. L'inscription d'Isyllos serait alors à dater précisément du moment de la préparation de la seconde tentative d'Areus, ce qui implique - nouvelle supposition de placer cette dernière après ou au moment de l'invasion galate. Toutefois, après avoir tenté de montrer qu'Isyllos aurait essayé de vanter la supériorité d'Asklépios sur d'autres dieux dont Apollon - Asklépios vient seul à bout des Macédoniens ! -, l'A. revient à une plus grande prudence en rappelant in fine qu'il n'est pas certain que l'on puisse établir de lien direct entre l'intervention d'Asklépios et celle d'Apollon dans le cadre de l'invasion galate, ce sur quoi on ne peut que la suivre.

Sur l'ensemble du chapitre relatif aux questions de datation, on peut se laisser convaincre... ou montrer quelques réserves. Si les sympathies philo-laconiennes d'Isyllos ne font pas de doute, on pourra hésiter à mettre nécessairement les notions qui les expriment en relation directe avec l'expédition d'Areus en Phocide, tant elles appartiennent à une phraséologie répandue. En outre, dans la lecture qui est proposée, force est de reconnaître que l'appel aux Athéniens apparaît en quelque sorte crypté, ce qui convient mal à un texte de propagande et à un appel à mener la lutte contre un ennemi commun; ajoutons que les interventions divines au combat, sous des formes multiples dont l'épiphanie n'est qu'un aspect, ne se limitent pas aux guerres médiques et les «parallèles» dits de «situation» qui sont proposés ne sont pas suffisants, ni pour évoquer une "mise en scène marathonienne » de la rencontre du jeune Isyllos avec Asklépios, ni pour affirmer que le passage s'adresse «avant tout à un public athénien ». La volonté de proposer à tout prix une date précise conduit, de manière parfois excessive, à substituer aux données historiques manquantes des raisonnements fondés sur des analogies, des «parallèles » ou des «liens » dont on ne parvient pas à voir par quel processus historique ils se constituent.

Le dernier chapitre intitulé «L'Asclépios d'Isyllos » commence par une présentation des différentes versions de la naissance d'Asklépios destinée à faire ressortir les changements qu'Isyllos introduit dans la partie du péan consacrée à la généalogie de la divinité. On peut alors observer comment Isyllos, non sans un certain dialogue avec la tradition, fait avant tout d'Asklépios un Épidaurien, né à Épidaure, et dont les ancêtres à l'exception d'Apollon sont des Épidauriens. L'inscription d'Isyllos apparaît, dans un contexte de rivalités entre plusieurs Asklépieia, porteuse d'une propagande visant à établir la préséance du sanctuaire d'Épidaure sur les autres. En cela, il rejoint l'une des finalités des récits des iamata soumis à la lecture des fidèles venant accomplir le rite de l'incubation et avec lesquels l'inscription d'Isyllos présente quelques similitudes, notamment parce que le dieu qui y est mis en scène y apparaît comme dieu guérisseur détenteur également d'un pouvoir oraculaire. Il reste que l'Asklépios d'Isyllos offre aussi l'image d'un dieu qui, revêtu d'une armure d'or brillante, quitte son sanctuaire pour aller au secours de Sparte menacée par Philippe, ce qui lui vaut alors de recevoir l'épiclèse Sôter. Donnant à l'épiclèse une acception politique et rangeant le dieu, à l'instar d'Apollon, du côté des divinités protectrices des 
régimes aristocratiques, l'A. propose de voir un parallèle métaphorique (p. 322-323) entre la santé des hommes et celle d'une constitution comme en témoignerait, selon

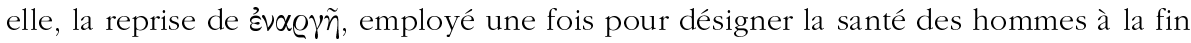
du péan (1. 55-56), l'autre fois pour qualifier les paroles du dieu annonçant sa décision d'aller au secours de Sparte et de sa constitution (1. 67). Si le caractère politique de la fonction dévolue alors à la divinité ne fait pas de doute, sans doute conviendrait-il, pour mieux l'expliquer, de ne pas partir de l'idée a priori qu'Asklépios est « un dieu apparemment si étranger à la cité » mais, au contraire, de s'interroger d'emblée sur les relations que ce dernier entretient, à Épidaure comme ailleurs (et notamment à Messène), avec les communautés civiques.

L'ouvrage comprend en annexe un choix de treize lois sacrées (textes et traductions) correspondant à celles qui ont été utilisées pour établir la comparaison avec celle qui est formulée dans l'inscription d'Isyllos, ainsi que les textes relatifs à la naissance d'Asklépios. S'y ajoute un long appendice de plus d'une trentaine de pages intitulé « $\sum \omega \tau$ iń : Démétrios Poliorcète, Ptolémée Ier et Asclépios »; l'A. cherche à établir un « rapprochement » entre l'Asklépios d'Isyllos et les souverains hellénistiques en question, à qui l'épithète a été attribué. La question a été posée dans le cours du dernier chapitre (p. 326) et l'on voit mal pourquoi son traitement est alors rejeté hors du texte principal. On trouve alors un certain nombre de considérations sur le culte rendu aux souverains hellénistiques, le texte, traduit et commenté, de l'hymne des Athéniens en l'honneur de Démétrios Poliorcète ainsi que le passage de l'inscription se rapportant à l'épiphanie d'Athéna Lindia qui aurait conduit à l'intervention de Ptolémée I ${ }^{\text {er }}$ à la fin du siège de Rhodes par Démétrios. Des points communs sont alors recherchés, en particulier entre les modalités de l'intervention du dieu dans l'inscription d'Isyllos et celles de l'action des souverains, ce qui permet d'aboutir à la conclusion que le texte d'Isyllos reprend certains éléments constitutifs de la propagande utilisée par les souverains hellénistiques. Une bibliographie et des indices complètent cet ouvrage.

En dépit de pages parfois moins convaincantes et qui, à tout le moins, ouvrent le champ de la discussion, l'ensemble constitue d'ores et déjà l'ouvrage de référence sur l'inscription d'Isyllos d'Épidaure tout en brossant un portrait d'Asclépios en " habit de citoyen », au cour des relations entre religion et politique, une place que les études antérieures avaient souvent ignorée.

Pierre Sineux

(Université de Caen)

Ilias N. ARnaoutoglou, Thusias heneka kai sunousias. Private religious associations in Hellenistic Athens, Athens, Academy of Athens, 2003. 1 vol. $17 \times 24$ cm, 231 p. (Academy of Athens. Yearbook of the Research Centre for the History of Greek Law, 37, supplement 4). ISBN : 960-404-034-0.

The title of this book comprises two phrases, one in ancient Greek, the other one in modern English. This dichotomy reflects a basic characteristic of the subject: it can be adequately described and perceived only within the framework of the Greek culture, whereas modern definitions and comparisons, indispensable its discussion, are always inaccurate, and often misleading. The author (henceforth A.) is completely aware of this problem. The book starts with a warning that modern word usage and ideas about cult groups in the contemporary world are inappropriate for Classical and Hellenistic Athens. Towards the end of his study, the A. demonstrates that "the modern 